Global Journal of Pure and Applied Mathematics.

ISSN 0973-1768 Volume 11, Number 2 (2015), pp. 875-886

(C) Research India Publications

https://dx.doi.org/10.37622/GJPAM/11.2.2015.875-886

\title{
On Regular $\boldsymbol{\beta}^{\wedge}$ - Generalized Closed Sets in Topological Spaces
}

\author{
Nitin Bhardwaj, Harpreet Kaur, B. P. Garg \\ Nitin Bhardwaj is currently pursuing his PhD in Mathematics, \\ NIMS, Jaipur, Rajasthan India \\ Harpreet Kaur is student in Lovely Professional University, Punjab, India, \\ B. P. Garg is currently working in Punjab Technical University, \\ Jalandhar, Punjab, India
}

\begin{abstract}
In this paper, a new class of sets called regular $\beta^{\wedge}$ - generalized closed (briefly $r \beta^{\wedge} g$-closed) sets in topological spaces is introduced and properties of this class of sets are studied. A subset $A$ of a topological space $(X, \tau)$ is called $r \beta^{\wedge}$-generalized closed (briefly $r \beta^{\wedge} g$-closed) set if $\operatorname{cl}(\operatorname{int}(\operatorname{cl}(A))) \subseteq U$ whenever $A \subseteq U$ and $U$ is $\beta$-open in $X$. This new class of sets lies between the class of regular closed (briefly $r$-closed) sets and $\beta^{\wedge}$ - generalized closed (briefly $\beta^{\wedge} g$-closed) sets.
\end{abstract}

\section{Mathematics Subject Classification: 54A05}

Keywords: $\beta$ - open, $r$-closed, $\beta^{\wedge} g$-closed sets

\section{INTRODUCTION}

The generalization of closed sets in the study of topological spaces is a fundamental concept. In 1970, N. Levine introduced the concept of generalized closed sets in topological spaces as a subset $A$ of $X$ is called generalized closed (briefly $g$-closed) set [20] $\operatorname{iff} c l(A) \subseteq U$ whenever $A \subseteq U$ and $U$ is open. This generalization of closed sets has led to several new and interesting concepts along with their contributions to the theories of separation axioms and generalizations of continuity. After the introduction of generalized closed sets there are many research papers which deal with different types of generalized closed sets. In 1990, S.P. Arya and T. M. Nour defined generalized semi open sets, generalized semi closed sets [25].

In 1993, N. Palanilnappan and K. ChandrasekharaRao introduced regular generalized 
closed [21] (briefly $r g$-closed ) sets and studied their properties.In 2000, A. Pushpalatha introduced new class of closed sets called weakly closed (briefly $w$ closed) sets along with its properties. In 2007, S.S. Benchalli and R. S. Wali introduced the new class of the sets called regular weakly closed [26] (briefly $r w$ closed) sets in topological spaces. In 2012, S. Mishra, N. Bhardwaj and V. Joshi introduced the new class of closed sets called regular generalized weakly closed [28] (briefly $r g w$-closed) sets and also generalized pre-regular weakly closed [29] (brieflygprw-closed) sets in topological spaces. Andeijevic introduced semi-preopen sets which are also known as $\beta$-open sets. K. Kannan, N. Nagaveni introduced the concept $\beta^{\wedge}$-generalized closed sets and open sets [14] in which properties of $\beta^{\wedge}$ generalized closed sets and some basic properties of $\beta^{\wedge}$-generalized open are discussed. In this paper we define a new generalization of closed sets called $\beta^{*}$ generalized closed (briefly $\beta^{*} g$-closed) set lies between regular closed (briefly $r$ closed) set and $\beta^{\wedge}$-generalized closed (briefly $\beta^{\wedge} g$-closed) set.

Throughout this paper, the space $(X, \tau)$ (or simply $X$ ) always means a topological space on which no separation axioms are assumed unless explicitly stated. For a subset $A$ of a space $X, \operatorname{cl}(A), \operatorname{int}(A)$ and $A^{c}$ denote the closure of $A$, interior of $A$ and complement of $A$ in $X$ respectively.

\section{Preliminaries}

\section{Definition 2.1}

A subset $A$ of $X$ is called generalized closed (briefly $g$-closed)[20] set if $\operatorname{cl}(A) \subseteq U$ whenever $A \subseteq U$ and $U$ is open.

\section{Definition 2.2}

A subset $A$ of $X$ is called regular open [16] (briefly $r$-open) set if $A=\operatorname{int}(\operatorname{cl}(A))$ and regular closed (briefly $r$-closed) set if $A=\operatorname{cl}(\operatorname{int}(A))$.

\section{Definition 2.3}

A subset $A$ of $X$ is called pre-open set [1] if $A \subseteq \operatorname{int}(\operatorname{cl}(A))$ and pre-closed set if $\operatorname{cl}(\operatorname{int}(A)) \subseteq A$.

\section{Definition 2.4}

A subset $A$ of $X$ is called semi-open set [19] if $A \subseteq \operatorname{cl}(\operatorname{int}(A))$ and semi-closed set if $\operatorname{int}(\operatorname{cl}(A)) \subseteq A$.

\section{Definition 2.5}

A subset $A$ of $X$ is called $\alpha$ - open set [23] if $A \subseteq \operatorname{int}(\operatorname{cl}(\operatorname{int}(A)))$ and $\alpha$ - closed if $\operatorname{cl}(\operatorname{int}(\operatorname{cl}(A)) \subseteq A$.

\section{Definition 2.6}

Asubset $A$ of $X$ is called semi-preopen [6](= $\beta$-open[17]) if $A \subseteq \operatorname{cl}(\operatorname{int}(\operatorname{cl}(A)))$ and semi-preclosed if $\operatorname{int}(\operatorname{cl}(\operatorname{int}(A))) \subseteq A$. 


\section{Definition 2.7}

A subset $A$ of a space $(X, \tau)$ is called regular semi- open [5] if there is a regular semiopen set $U$ such that $U \subset A \subset \operatorname{cl}(U)$. The family of all open sets of $X$ is denoted by $R S O(X)$.

\section{Definition 2.8}

A subset $A$ of a space $(X, \tau)$ is said to be semi-regular open [7] if it is both semi-open and semi-closed.

\section{Definition 2.9}

A subset $A$ of a space $(X, \tau)$ is said to be semi-generalized closed (briefly $s g$-closed) [24] if $\operatorname{scl}(A) \subseteq U$ whenever $A \subseteq U$ and $U$ is semi-open in $X$.

\section{Definition 2.10}

A subset $A$ of a space $(X, \tau)$ is said to be generalized semi-closed (briefly $g s$ - closed) [25] if $\operatorname{scl}(A) \subseteq U$ whenever $A \subseteq U$ and $U$ is open in $X$.

\section{Definition 2.11}

A subset $A$ of a space $(X, \tau)$ is said to be generalized $\alpha$-closed (briefly $g \alpha$-closed) [10] if $\alpha-\operatorname{cl}(A) \subseteq U$ whenever $A \subseteq U$ and $U$ is $\alpha$-open in $X$.

\section{Definition 2.12}

A subset $A$ of a space $(X, \tau)$ is said to be $\alpha$-generalized closed (briefly $\alpha g$-closed) [9] if $\alpha-\operatorname{cl}(A) \subseteq U$ whenever $A \subseteq U$ and $U$ is open in $X$.

\section{Definition 2.13}

A subset $A$ of a space $(X, \tau)$ is said to begeneralized semi-preclosed (briefly $g s p$ closed) [12] if $\operatorname{spcl}(A) \subseteq U$ whenever $A \subseteq U$ and $U$ is open in $X$.

\section{Definition 2.14}

A subset $A$ of a space $(X, \tau)$ is said to be regular generalized closed (briefly $r g$ closed) [21] if $\operatorname{cl}(A) \subseteq U$ whenever $A \subseteq U$ and $U$ is regular open in $X$.

\section{Definition 2.15}

A subset $A$ of a space $(X, \tau)$ is said to be generalized preclosed (briefly $g p$-closed) [11] if $\operatorname{pcl}(A) \subseteq U$ whenever $A \subseteq U$ and $U$ is open in $X$.

\section{Definition 2.16}

A subset $A$ of a space $(X, \tau)$ is said to begeneralized pre regular closed (briefly gprclosed) [31] whenever $\operatorname{pcl}(A) \subseteq U$ whenever $A \subseteq U$ and $U$ is regular open in $X$.

\section{Definition 2.17}

A subset $A$ of a space $(X, \tau)$ is said to beweakly generalized closed (briefly $w g$ closed) [22] if $\operatorname{cl}(\operatorname{int}(A)) \subseteq U$ whenever $A \subseteq U$ and $U$ is open in $X$. 


\section{Definition 2.18}

A subset $A$ of a space $(X, \tau)$ is said to be strongly generalized closed (briefly $g^{*}$ closed) [] if $\operatorname{cl}(A) \subseteq U$ whenever $A \subseteq U$ and $U$ is g-open in $X$.

\section{Definition 2.19}

A subset $A$ of a space $(X, \tau)$ is said to beweakly closed (briefly $w$-closed) [2] if $\operatorname{cl}(A) \subseteq U$ whenever $A \subseteq U$ and $U$ is semi-open in $X$.

\section{Definition 2.20}

A subset $A$ of a space $(X, \tau)$ is said to be mildly generalized closed (briefly mildly $g$ closed) [13] if $\operatorname{cl}(\operatorname{int}(A)) \subseteq U$ whenever $A \subseteq U$ and $U$ is g- open in $X$.

\section{Definition 2.21}

A subset $A$ of a space $(X, \tau)$ is said to besemi weakly generalized closed (briefly $s w g$ closed) [22] if $\operatorname{cl}(\operatorname{int}(A)) \subseteq U$ whenever $A \subseteq U$ and $U$ is semi- open in $X$.

\section{Definition 2.22}

A subset $A$ of a space $(X, \tau)$ is said to be regular weakly generalized closed (briefly $r w g$-closed) [22] if $\operatorname{cl}(\operatorname{int}(A)) \subseteq U$ whenever $A \subseteq U$ and $U$ is regular open in $X$.

\section{Definition 2.23}

A subset $A$ of a space $(X, \tau)$ is said to be regular weakly closed (briefly $r w$-closed) [26] if $\operatorname{cl}(A) \subseteq U$ whenever $A \subseteq U$ and $U$ is regular semi-open in $X$. We denote the set of all $r$-closed sets in $X$ by $R W C(X)$.

\section{Definition 2.24}

A subset $A$ of a space $(X, \tau)$ is said to be $\beta^{\wedge}$-generalized closed (briefly $\beta^{\wedge} g$ closed) [14] if $\operatorname{cl}(\operatorname{int}(\operatorname{cl}(A))) \subseteq U$ whenever $A \subseteq U$ and $U$ is open in $X$.

\section{Remark:}

The complements of the above mentioned closed sets are their respective open sets.

\section{On $\boldsymbol{r} \boldsymbol{\beta}^{\wedge}$-generalized closed sets}

In this section we introduce the concept of $r \beta^{\wedge}$-generalized closed set (briefly $r \beta^{\wedge} g$ closed) which lies between regular closed (briefly $r$-closed) set and $\beta^{\wedge}$-generalized closed (briefly $\beta^{\wedge} g$-closed) set and some of their basic properties.

\section{Definition 3.1}

A subset $A$ of a topological space $(X, \tau)$ is called $r \beta^{\wedge}$-generalized closed (briefly $r \beta^{\wedge} g$-closed) set if $\operatorname{cl}(\operatorname{int}(\operatorname{cl}(A))) \subseteq U$ whenever $A \subseteq U$ and $U$ is $\beta$-open in $X$.

\section{Theorem 3.2}

Every open set is $\beta$-open in space $X$. 


\section{Proof:}

Let $A$ be an arbitrary open set in $X$ i.e. $A=\operatorname{int}(A)$ and we know $A \subseteq \operatorname{cl}(A)$ i.e. $A=$ $\operatorname{int}(A) \subseteq \operatorname{int}(\operatorname{cl}(A))$. Then $\operatorname{cl}(A) \subseteq \operatorname{cl}(\operatorname{int}(\operatorname{cl}(A)))$. So by def. 2.6, $A$ is an $\beta$-open set.

\section{Remark 3.3}

The converse of this theorem need not to be true as seen from the following example.

\section{Example 3.4}

Consider $X=\{a, b, c, d\}$ be a space with the topology $\tau=$ $\{\phi, X,\{a\},\{b\},\{a, b\},\{a, b, c\}\}$. Here $A=\{b, d\}$ is $\beta$-open but not open in $X$.

\section{Theorem 3.5}

Every $r$-closed set in $X$ is $r \beta^{\wedge} g$-closed set in $X$.

\section{Proof:}

Let $A$ be regular closed set in the space $X$. Suppose $A \subset U$ and $U$ be $\beta$-open in $X$. Follow from Stone [16] and by def. 2.2, we have $A=\operatorname{cl}(\operatorname{int}(\operatorname{cl}(A)))$. Since $A \subset U$, so that it implies $\operatorname{cl}(\operatorname{int}(\operatorname{cl}(A))) \subset U$. Hence $\operatorname{cl}(\operatorname{int}(\operatorname{cl}(A))) \subset U$ whenever $A \subset U$ and $U$ is $\beta$-open.Therefore $A$ is $r \beta^{\wedge} g$-closed set in $X$.

\section{Remark 3.6}

The converse of this theorem need not to be true as seen from the following example.

\section{Example 3.7}

Consider $X=\{a, b, c, d\}$ be a space with the topology $\tau=$ $\{\phi, X,\{a\},\{b\},\{a, b\},\{a, b, c\}\}$. Here $A=\{c, d\}$ is $r \beta^{\wedge} g$-closed but not regular closed set in $X$.

\section{Theorem 3.8}

Every $r \beta^{\wedge} g$-closed set in space $X$ is $\beta^{\wedge} g$-closed set.

\section{Proof:}

Let $A$ be an arbitrary $r \beta^{\wedge} g$-closed set in space $X$. Suppose $A \subset U$ and $U$ is open in $X$. Since every open set is $\beta$-open in $X$. So we can say that $A \subset U$ and $U$ be $\beta$-open in $X$. Hence by definition 3.1, we have $\operatorname{cl}(\operatorname{int}(\operatorname{cl}(A))) \subset U$ whenever $A \subset U$. Finally $A$ is $\beta^{\wedge} g$-closed set.

\section{Remark 3.9}

The converse of this theorem need not to be true as seen from the following example.

\section{Example 3.10}

Consider $X=\{a, b, c, d\}$ be a space with the topology $=\{\phi, X$, $\{a\},\{b\},\{a, b\},\{a, b, c\}\}$. Here $A=\{a, d\}$ is $\beta^{\wedge} g$-closed set but not $r \beta^{\wedge} g$-closed set 
in $X$.

\section{Remark 3.11}

The following examples are shows that $r \beta^{\wedge} \mathrm{g}$-closed sets are independent of rw-closed sets, $g^{*}$-closed, mildly g-closed sets, g-closed sets, gp-closed sets, $\theta$-geneeralized closed, $\delta$-generalized closed and regular semi-closed sets.

\section{Example 3.12}

Let $X=\{a, b, c, d\}$ be a space with the topology $=\{\phi, X,\{a\},\{b\},\{a, b\},\{a, b, c\}\}$. Then

1. Closed sets in $(X, \tau)$ are $\{\phi, X,\{d\},\{c, d\},\{a, c, d\},\{b, c, d\}\}$.

2. $r$-closed sets in $(X, \tau)$ are $\{\phi, X,\{a, c, d\},\{b, c, d\}\}$.

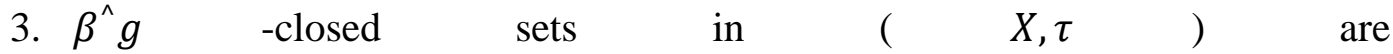
$\{\phi, X,\{c\},\{d\},\{a, d\},\{b, d\},\{c, d\},\{a, b, d\},\{a, c, d\},\{b, c, d\}\}$

4. $r \beta^{\wedge} g$-closed sets in $(X, \tau)$ are $\{\phi, X,\{c\},\{d\},\{c, d\},\{a, c, d\},\{b, c, d\}\}$.

5. $\mathrm{R} w$-closed sets in $w(X, \tau)$ are $\{\phi, X,\{d\},\{c, d\},\{a, b\},\{a, b, c\},\{a, b, d\},\{a, c, d\},\{b, c, d\}\}$.

6. $g^{*}$-closed sets in $(x, \tau)$ are $\{\phi, X,\{d\},\{c, d\},\{a, d\},\{b, d\},\{a, b, d\},\{a, c, d\},\{b, c, d\}\}$.

7. Mildly $g$-closed sets in $(X, \tau)$ are $\{\phi, X,\{d\},\{c, d\},\{a, d\},\{b, d\},\{a, b, d\},\{a, c, d\},\{b, c, d\}\}$.

8. $g$-closed sets in $\left(\begin{array}{r}X, \tau) \text { are }\end{array}\right.$ $\{\phi, X,\{d\},\{c, d\},\{a, d\},\{b, d\},\{a, b, d\},\{a, c, d\},\{b, c, d\}\}$.

9. $G p$-closed sets in $(X, \tau)$ are $\{\phi, X,\{c\},\{d\},\{c, d\},\{a, d\},\{b, d\},\{a, b, d\}\}$.

10. Regular semi-closed sets in ( $X, \tau)$ are $\{\phi, X,\{a\},\{b\},\{a, c\},\{a, d\},\{b, c\},\{b, d\},\{a, c, d\},\{b, c, d\}\}$.

11. $\theta$-generalized closed sets in $(X, \tau)$ are $\{\phi, X,\{d\},\{c, d\},\{a, b\},\{a, b, c\},\{a, b, d\},\{a, c, d\},\{b, c, d\}\}$.

12. $\delta$-generalized closed sets in ( $X, \tau)$ $\operatorname{are}\{\phi, X,\{d\},\{c, d\},\{a, d\},\{b, d\},\{a, c, d\},\{a, b, d\},\{b, c, d\}$

13.rgw -closed sets in $(X, \tau)$ are $\{\phi, X,\{c\},\{d\},\{c d\},\{a, b\},\{a, b, c\},\{a, b, d\},\{a, c, d\},\{b, c, d\}\}$.

14. gprw -closed sets in $(X, \tau)$ are $\{\phi, X,\{d\},\{a, b\},\{c, d\},\{a, b, c\},\{a, b, d\},\{a, c, d\},\{b, c, d\}\}$.

\section{Theorem 3.13}

The union of two $r \beta^{\wedge} \mathrm{g}$-closed subsets of $X$ is also $r \beta^{\wedge} \mathrm{g}$-closed subset of $X$.

\section{Proof:}

Assume that $A$ and $B$ arer $\beta^{\wedge}$ g-closed set in $X$. Let $U$ be the $\beta$-open set in $X$ such that $A \cup B \subset U$. Then $A \subset U$ and $B \subset U$. Since $A$ and $B$ are $r \beta^{\wedge}$ g-closed, $\operatorname{cl}(\operatorname{int}(\operatorname{cl}(A))) \subset U$ and $\operatorname{cl}(\operatorname{int}(\operatorname{cl}(B))) \subset U$. Hence $\operatorname{cl}(\operatorname{int}(\operatorname{cl}(A \cup B)))=$ $\operatorname{cl}(\operatorname{int}(\operatorname{cl}(A))) \cup \operatorname{cl}(\operatorname{int}(\operatorname{cl}(B))) \subset U$. This implies that $\operatorname{cl}(\operatorname{int}(\operatorname{cl}(A \cup B))) \subset U$. Therefore $A \cup B$ is $r \beta^{\wedge} \mathrm{g}$-closed set in $X$. 


\section{Theorem 3.16}

If subset $A$ of $X$ is $r \beta^{\wedge}$ g-closed set in $X$. Then $c l(\operatorname{int}(\operatorname{cl}(A)))-A$ does not contain any non empty $\beta$-open set in $X$.

\section{Proof:}

Suppose that $A$ is $r \beta^{\wedge}$ g-closed set in $X$. We prove the result by contradiction. Let $U$ be $\beta$-open set such that $\operatorname{cl}(\operatorname{int}(\operatorname{cl}(A)))-A \supset U$ and $U \neq \phi$. Now $U \subset$ $\operatorname{cl}(\operatorname{int}(\operatorname{cl}(A)))-A$. Therefore $U \subset X-U$. Since $U$ is $\beta$-open set, $X-U$ is also $\beta$ open in $X$. Since $A$ is $r \beta^{\wedge}$ g-closed sets in $X$, by definition 3.1, we have $c l(\operatorname{int}(\operatorname{cl}(A))) \subset X-U$. So $U \subset X-\operatorname{cl}(\operatorname{int}(\operatorname{cl}(A)))$. Also $U \subset \operatorname{cl}(\operatorname{int}(\operatorname{cl}(A)))$. Therefore $U \subset \operatorname{cl}(\operatorname{int}(\operatorname{cl}(A))) \cap(X-\operatorname{cl}(\operatorname{int}(\operatorname{cl}(A)))=\phi$. This shows that $U=$ $\phi$ which a contradiction is. Hence $\operatorname{cl}(\operatorname{int}(\operatorname{cl}(A)))-A$ does not contain any non empty $\beta$-open set in $X$.

\section{Remark 3.17}

The converse of this theorem needs not to be true as seen from the following example.

\section{Example 3.18}

If $\operatorname{cl}(\operatorname{int}(\operatorname{cl}(A)))-A$ does not contains any non empty open set in $X$, then $A$ need not to be $r \beta^{\wedge}$ g-closed. Consider $X=\{\mathrm{a}, \mathrm{b}, \mathrm{c}\}$ with the topology $\tau=\{\phi, X,\{\mathrm{a}\},\{\mathrm{a}$, b $\}\}$ and $A=\{\mathrm{a}, \mathrm{b}\}$. Then $\operatorname{cl}(\operatorname{int}(\operatorname{cl}(A)))-A=X-\{a, b\}=\{c\}$ does not contain any non empty open set, but $A$ is not a $r \beta^{\wedge} \mathrm{g}$-closed set in $X$.

\section{Theorem 3.19}

For an element $x \in X$, the set $X-\{x\}$ is $r \beta^{\wedge}$ g-closed or $\beta$-open.

\section{Proof:}

Suppose $X-\{x\}$ is not $\beta$-open.This means the only choice of $\beta$-open set containing $X-\{x\}$ is $X$. This implies $c l(\operatorname{int}(\operatorname{cl}(X)))-\{x\} \subset X$. Hence $X-\{x\}$ is an $r \beta^{\wedge} \mathrm{g}$ closed set in $X$.

\section{Theorem 3.20}

If $A$ is an $r \beta^{\wedge}$ g-closed subset in $X$ s.t $A \subset B \subset \operatorname{cl}(A)$, then $B$ is an $r \beta^{\wedge}$ g-closed set in $X$.

\section{Proof:}

Let $A$ be an arbitrary $r \beta^{\wedge}$ g-closed set in $X$ s.t $A \subset B \subset \operatorname{cl}(A)$. Let $U$ be a $\beta$-open set of $X$ s.t. $B \subset U$. Then $A \subset U$. Since $A$ is $r \beta^{\wedge}$ g-closed so, we have $\operatorname{cl}(A) \subset U$. Now $\operatorname{cl}(B) \subset \operatorname{cl}(\operatorname{cl}(A))=\operatorname{cl}(A) \subset U$. Therefore $B$ is an $r \beta^{\wedge}$ g-closed set in $X$. 


\section{Remark3.21}

The converse of this theorem needs not to be true as seen from the following example.

\section{Example 3.22}

Consider the topological space $X=\{a, b, c\}$ with the topology $\tau=\{\phi, X,\{b\},\{b, c\}\}$. Let $A=\{\mathrm{a}\}$ and $\mathrm{B}=\{\mathrm{a}, \mathrm{c}\}$. Then $A$ and $B$ arer $\beta^{\wedge} \mathrm{g}$-closed sets in $X$, but $A \subset B$ is not subset in $\operatorname{cl}(A)$.

\section{Remark3.15}

Now we see the relationship from the following figure between the different generalizations of closed sets and $r \beta^{\wedge} g$-closed set in topological spaces.

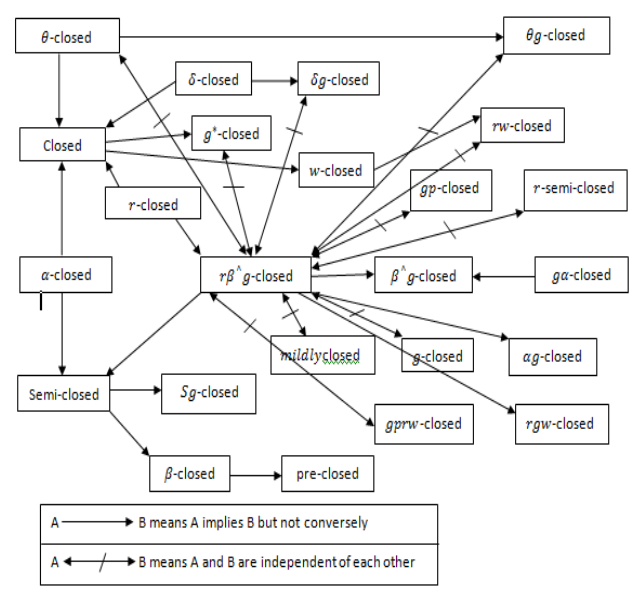

Definition 3.23

The intersection of all $\beta$-open subsets of $(X, \tau)$ containing $A$ is called $\beta$-kernel of $A$ and is denoted by $\beta \operatorname{ker}(A)$.

\section{Remark3.24}

Let $X$ be a topological space and $A$ be a subset of $X$. If $A$ is $\beta$-open in $X$, then $\beta \operatorname{ker}(A)=A$, but conversely not true.

\section{Remark3.25}

For any subset $A$ of $(X, \tau), A \subset \beta \operatorname{ker}(A)$.

\section{Theorem 3.26}

A subset $A$ of $(X, \tau)$ is $r \beta^{\wedge} g$-closed if and only if $\operatorname{cl}(\operatorname{int}(\operatorname{cl}(A))) \subset \beta \operatorname{ker}(A)$.

\section{Proof:}

Suppose that $A$ is $r \beta^{\wedge} g$-closed. $\operatorname{cl}(A) \subset U$, whenever $A \subset U$ and $U$ is $\beta$-open. Let $x \in \operatorname{cl}(A)$. Suppose $x \notin \beta \operatorname{ker}(A)$; then there is a $\beta$-open set $U$ containing $A$ such 
that $x$ is not in $U$. Since $A$ is $r \beta^{\wedge} g$-closed. We have $x$ is not $\operatorname{cl}(A)$, which is a contradiction. Hence $x \in \beta \operatorname{ker}(A)$ and $\operatorname{cl}(A) \subset \beta \operatorname{ker}(A)$. Conversely, let $\operatorname{cl}(A) \subset$ $\beta \operatorname{ker}(A)$. If $U$ is any $\beta$-open set containing $A$, then $\beta \operatorname{ker}(A) \subset U$. That is $A$ is $r \beta^{\wedge} g$ closed in $X$.

On $r \beta^{\wedge}$ - generalized open sets and $r \beta^{\wedge}$ - generalized neighborhoods in topological spaces

In this section, we introduce and study $r \beta^{\wedge}$-generalized open sets (briefly $r \beta^{\wedge} g$-open) and $r \beta^{\wedge}$-generalized neighborhoods (briefly $r \beta^{\wedge} g$-nhbd) in topological spaces and obtain some of their properties.

\section{Definition 4.1}

A subset $A$ in $X$ is called is called $r \beta^{\wedge}$-generalized open sets (briefly $r \beta^{*} g$-open) in $X$ if $A^{c}$ is $r \beta^{\wedge} g$-closed in $X$. We denote the family of all $r \beta^{\wedge} g$-open sets in $X$ by $r \beta^{\wedge} O(X)$.

\section{Theorem 4.2}

If $A$ and $B$ arer $\beta^{\wedge} g$-open sets in topological space $X$. Then $A \cap B$ is also $r \beta^{\wedge} g$-open set in $X$.

\section{Proof:}

Let $A$ and $B$ arer $\beta^{\wedge} g$-open sets in a space $X$. Then $A^{c}$ and $B^{c}$ are $r \beta^{\wedge} g$-closed sets in $X$. By Theorem 3.13, $A^{c} \cup B^{c}$ is also $r \beta^{\wedge} g$-closed sets in $X$.That is $A^{c} \cup B^{c}=$ $(A \cap B)^{c}$ is also a $r \beta^{\wedge} g$-closed sets in $X$. Therefore $(A \cap B)$ is $r \beta^{\wedge} g$-open set in $X$.

\section{Remark 4.4}

For any $A \subset X, \beta \operatorname{int}(\operatorname{cl}(\operatorname{int}(\operatorname{cl}(A)))-A)=\phi$.

\section{Theorem 4.5}

If $A \subset X$ isr $\beta^{\wedge} g$-closed then $\operatorname{cl}(\operatorname{int}(\operatorname{cl}(A)))-A$ is $r \beta^{\wedge} g$-open set in a space $X$.

\section{Proof:}

Let $A$ be an arbitrary $r \beta^{\wedge} g$-closed. Let $U$ be $\beta$-open s.t $U \subset \operatorname{cl}(\operatorname{int}(\operatorname{cl}(A)))-A$. Then by Theorem 3.16, $U=\phi$. This showscl $(\operatorname{int}(\operatorname{cl}(A)))-A$ is $r \beta^{\wedge} g$-open.

\section{Remark 4.6}

$\operatorname{cl}(A)=X-\operatorname{int}(A)$

\section{Theorem 4.7}

If $\operatorname{int}(A) \subset B \subset A$ and $A$ is $r \beta^{\wedge} g$-open in $X$, then $B$ is $r \beta^{\wedge} g$-open in $X$. 


\section{Proof:}

Suppose that $\operatorname{int}(A) \subset B \subset A$ and $A$ is $r \beta^{\wedge} g$ open. Hence $X-A \subset X-B \subset C l(X-$ $A)$. Since $X-A$ is $r \beta^{\wedge} g$-closed. Then by Theorem 3.20, $X-B$ is $r \beta^{\wedge} g$-closed. Thus $B$ is $r \beta^{\wedge} g$-open.

\section{Definition 4.8}

Let $X$ be a topological space and let $x \in X$. A subset $N$ of $X$ is said to be a $r \beta^{\wedge} g-$ nhbd of $x$ iff there is $r \beta^{\wedge} g$-open set $G$ such that $x \in G \subset N$.

\section{Definition 4.9}

A subset $N$ of a space $X$, is called a $r \beta^{\wedge} g$-nhbd of $A \subset X$ iff there exists a $r \beta^{\wedge} g$-open set $G$ such that $A \subset G \subset N$.

\section{Theorem 4.10}

Every $\operatorname{nhbd} N$ of $x \in X$ is a $r \beta^{\wedge} g$-nhbd of $X$.

\section{Proof:}

Let $N$ be a nhbd of a point $x \in X$. To prove that $N$ is a $r \beta^{\wedge} g$-nhbd of $x$. By def.4.8, there exist an open set $G$ such that $x \in G \subset N$. As every open set is $r \beta^{\wedge} g$-open set $G$ such that $x \in G \subset N$. Hence $N$ is $\beta^{*} g$-nhbd of $X$.

\section{Remark 4.11}

In general, a $r \beta^{\wedge} g$-nhbd $N$ of $x \in X$ need not to be a nhbd of $x$ in $X$, as from the following example.

\section{Example 4.12}

Cosnider $X=\{a, b, c\}$ be a space with the topology $\tau=\{\phi, X,\{c\}\}$. The set $\{a, b\}$ is $r \beta^{\wedge} g$-nhbd of the point $b$, since the $r \beta^{\wedge} g$-open set $\{b\}$ is such that $b \in\{b\} \subset\{a, b\}$. However the set $\{a, b\}$ is not a nhbd of the point $b$, since no open set $G$ exists such that $b \in G \subset\{a, b\}$.

\section{References}

[1] A.S. Mashhour, M.E. Abd. El-Monsef and S.N. El-Deeb, On pre-continuous mappings and weak pre-continuous mappings, Proc Math, Phys. Soc. Egypt, 53(1982), 47-53.

[2] A. Pushpalatha, studies on generalization of mappings in topological spaces, $\mathrm{Ph}$. D. Thesis, Bharathiar University, Coimbatore, 2000.

[3] AHMAD AL-OMARI AND MOHD SALMI MD. NOORANI, On Generalized b-closed Sets, Bull Malays. Math. Sci. Soc. (2) 32(1) (2009), 1930 .

[4] A. K. Al-Obiadi, $\pi$-Generalized $b$-Closed Sets in Topological spaces, Ibn Al- Haithamj J. for Pure \& Appl Sci. 24(3)(2011). 
[5] D.E. Cameron, Properties of S-closed spaces, Proc. Amer Math. Soc., 72(1978), 961-970.

[6] DimitrijeAndrijeric, Semi-preopen sets, Mat. Vesnik, 38(1986), 24-32.

[7] G. Di Maio and T. Noiri, On s-closed spaces, Indian J. Pure Appl. Math., 18(3)(1987), 226-233.

[8] Govindappa Navalagi, Chandrashekarappa A. Sand S.V Gurushantanavar, ON GSPR- CLOSED SETS IN TOPOLOGICAL SPACES, International Journal of Mathematics an Computing Applications 2(2010), 51-58.

[9] H. Maki. R. Devi and K. Balachandran, Generalized $\alpha$-closed sets in topology, Bull. Fukuoka Uni. Ed. Part-III 42(1993), 13-21.

[10] H. Maki. R. Devi and K. Balachandran, Associated topologies of generalized $\alpha$-closed sets and $\alpha$-generalized closed sets, Mem. Sci. Kochi Univ. Ser. A. Math., 15(1994), 51-63.

[11] H. Maki, J. Umehara and T. Noiri, Every topological space is pre- $T_{\frac{1}{2}}, M e m$, Fac. Sci. Kochi. Univ. Ser. A. Math., 17(1996), 33-42.

[12] J. Dontchev, On generalizing semi-preopen sets, Mem. Fac Sci. Kochi. Uni. Ser. A. Math., 16(1995), 35-48.

[13] J. K. Park and J. H. Park, Mildly generalized closed sets, almost normal and mildly normal spaces, Chaos, Solitions and Fractals, 20(2004), 1103-1111.

[14] K. Kannan, N. Nagaveni, On $\beta^{\wedge}$-Generalized Closed Sets and Open Sets in Topological Spaces, Int. Journal of Math. Analysis, 6(2012), 2819-2828.

[15] K. Mariappa, S. Sekar, On Regular Generalized b-Closed Set, Int. Journal of Math. Analysis, 3(2013), 613-624.

[16] M.Stone, Application of theory of Boolean rings to general topology, Trans.Amer. Math. Soc., 41(1937), 374-481.

17] M.E. Abd. El-Monsef, S.N. El-Deeb and R.A. Mahmoud, $\beta$-open sets and $\beta$ continuous mappings, Bull. Fac. Sci. Assiut Univ., 12(1983), 77-90.

.[18] M. KHAN, T.NOIRI AND M. HUSSAIN, ON s* ${ }^{*}$-CLOSED SETS AND s*NORMAL SPACES, CODEN JNSMAC 1, 2 (2009), 31-41.

[19] N. Levine, Semi-open sets and semi-continuity in toplogical spaces, Amer. Math. Monthly 70(1963), 36-41.

[20] N. Levine, Generalized closed sets in toplogy, Rend. Circ. Mat. Palermo, 19(1970), 89-96.

[21] N. Palaniappan and K. C. Rao, Regular Generalized Closed sets, kyungpook math, J., 33(1993), 717-719.

[22] N. Nagaveni, Studies on Generalizations of Homeomorphisms in Topological Spaces, Ph. D. Thesis, Bharathiar University, Coimbatore, 1999.

[23] O. Njastad, On some classes of nearly open sets, Pacific J. Math., 15(1965), 961-970.

[24] P. Bhattacharya and B. K. Lahiri, Semi-generalized closed sets in topology, Indian J. Math., 29(1987), 226-233.

[25] S.P. Arya and T.M. Nour, Characterizations of s-normal spaces, Indian J. Pure App. Math, 21(1990), 717-719.

[26] S. S. Benchalli and R. S. Wali, On RW-closed sets in topological spaces, 
Ph.D. Thesis, BullMalaysian. Math.Sci.Soc. (2) 30(2) (2007), 99-110.

[27] SharmisthaBhattacharya(Halder ), On Generalized Regular Closed Sets, Int J. Contemp. Math. Sciences, 3(2011), 145-152.

[28] S. Mishra, N. Bhardwaj and V. Joshi, On Regular Generalized(rgw)-Closed Sets in Topological Spaces, Int. Journal of Math. Analysis 7(2012), 19391952.

[29] S. Mishra, V. Joshi and N. Bhardwaj, On Generalized Pre Regular Weakly (gprw) -Closed Sets in Topological Spaces, International Mathematical Forum 6(2012), 1981-1992.

[30] S. Muthuvel, R. Parimelazhagan, $b^{*}$-Closed Sets in Topological Spaces, Int. Journal of Math. Analysis, 6 (2012), 2317-2323.

[31] Y. Gnanambal, On generalized preregular closed sets in topological spaces, Indain J. PureApp. Math., 28( 1997), 351-360. 